\title{
METHYLPHENIDATE FOR BIPOLAR DEPRESSION
}

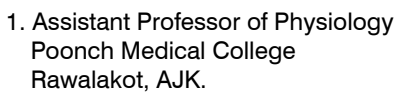

Correspondence Address: Muhammad Sajid Mehmood Assistant Professor of Physiology Poonch Medical College Rawalakot, AJK.

Article received on: 29/04/2017

Accepted for publication: 15/07/2017

Received after proof reading: 08/09/2017

\begin{abstract}
Muhammad Sajid Mehmood'
ABSTRACT: There is close association between Bipolar Disease (BPD) and Attention Deficit Hyperactivity Syndrome (ADHD). It has been reported that patients who suffer ADHD in childhood are more prone to BPD in adult life. So, there seems to be a same pathophysiology of both the diseases. Bipolar depression is one of most difficult challenge for psychiatrist to deal with; because of inconsistent results of available drugs authorized for use in BPD. Methylphenidate (MPH) is drug of choice in ADHD but not used in bipolar depression. MPH is considered contraindicated in BPD because it can switch on mania in BPD patients. This case report is written, based on the effects of MPH seen in this specific case along with other antidepressant in BPD. MPH may serve as a miracle for BPD patient and may reduce their suffering to a great extent.
\end{abstract}

Key words: Bipolar Depression, Methylphenidate, Antidepressant, Attention Deficit Hyperactivity Syndrome (ADHD).

Article Citation: Mehmood MS. Methylphenidate for bipolar depression. Professional Med $\mathrm{J}$ 2017;24(9):1450-1554. DOI: 10.17957/TPMJ/17.4011

\section{INTRODUCTION}

Bipolar depression (BPD) is one of a major unresolved task in the field of psychiatry. BPD is linked with substantial mortality and disability. The pathophysiology of BPD is unknown, which have resulted in failure of available treatment regimen. In BPD approximately half of life time is spent in morbid states in spite of using available treatments (antidepressants), even in compliant patients. BPD is exceptionally morbid condition with few treatments available. The pathophysiology of BPD needs to be explored so, potential relevant targets could be identified which may prove useful in developing new treatments regimen. ${ }^{1}$ Research evidence concerning efficacy and safety of commonly used antidepressant treatments for acute bipolar depression is very limited. Nevertheless, an updated meta-analysis indicated that overall efficacy was significantly greater with antidepressants than with placebotreatment and not less than was found in trials for unipolar major depression. Moreover, risks of non-spontaneous mood-switching specifically associated with antidepressant treatment are less than appears to be widely believed. The literature review encourages the superfluous efforts to test psychiatric medications which are acceptable and tolerable in BPD. The disease is linked with attention-deficit hyperactivity disorder, Bulimia nervosa, substance dependence and anxiety disorders. $^{2}$

Intravenous ketamine $(0.5 \mathrm{mg} / \mathrm{kg})$ produces rapid and long-lasting antidepressant effects, but is unworkable. Sublingual ketamine extracts better bioavailability $(\sim 30 \%)$ but the effects have been broad spectrum. Its antidepressant properties with high efficacy, rapid onset of action, good tolerability and minimal cost, allowing extended treatment as needed. ${ }^{3}$

In this case report the patient experience during combination therapy (i.e. venlafaxine, lamotrigine) along with methylphenidate and without methylphenidate is reported. The purpose of this case report is to describe the role of tablet 'methylphenidate' in a dose of $10 \mathrm{mg}$ taken in morning along with other antidepressants and mood stabilizer in the treatment of Bipolar depression which is a least studied drug for said purpose. 


\section{CASE PRESENTATION}

The patient suffered the problem at the age of 24 years when he was student of final year MBBS. Initially, the patient had severe depression according to Diagnostic and Statistical Manual of Mental Disorders (DCM-IV) classification. In order to get rid of his depression he was extremely motivated towards his religion and started prayers to his Lord to get rid of his depression. The subject had excessive weeping, feeling of anhedonia, lack of libido, low self esteem, suicidal ideation and worthlessness. The reason behind the depression was family issues and sudden anterolateral myocardial infarction of his father. Then, depression was followed by hypomania. There was excessive talking, pressure of speech, decreased sleep and getting fresh after only two hours sleep. The patient started preaching the religion and emphasizing people to respect parents, read holy-book and offer prayers. Initially, the patient resisted to visit any Psychiatrist because being a medical student, the patient thought that psychiatric medication will sedate him but his family forced him to visit a faith healer and then asked him to consult some doctor. Then, the patient finally agreed to consult his problem with the professor of Psychiatry and was prescribed with injection Flumazenil, tablet haloperidol, tablet clonazepam and tablet sodium valproate which the patient refused to take. However, he was forcefully given these medicine. As a result of medication, it was extremely difficult for the patient to carry on with his prayers. He could not wake up for 18 hours after taking these medicines. Next day, the patient suffered with severe torticollis. The patient rushed to a local clinic and asked the doctor to inject diazepam. The nurse injected diazepam intravenously and after 15 minutes the symptoms of neck stiffness and rolling of tongue disappeared. He was brought back home but after an hour extrapyramidal symptoms reappeared. That clinic was closed now and the patient bought the injection diazepam with great difficulty (being a controlled drug) and injected himself in deltoid muscle. He was taken to Accident and emergency department of a tertiary care hospital in the vicinity, where, a junior doctor gave him injection procyclidine and asked him to stop haloperidol and consult some psychiatrist next day.

Next day, the patient was taken to military hospital, where the professor of psychiatry prescribed him tablet resperidone and tablet sodium valproate twice daily (bd) in dose of $1 \mathrm{mg}$ and $500 \mathrm{mg}$ respectively. After a week of these medication he developed tremors in hands and severe arthralgia especially involving knee joints. He revisited the psychiatrist for these side effects but he was advised to continue the medication. After a month, the patient was put on tablet sodium valproate and tablet resperidone was stopped. The arthralgia settled but tremors continued and the patient was advised strictly not to quit the sodium valproate.

After six months of house job the patient quitted the sodium valproate which resulted in reappearance of depression. He was prescribed with tab Lithium and sertraline for depression. It resulted in excessive urination and dryness of mouth. He took this regimen for six to eight months but the depression was not relieved. Then, the subject started tablet Venlafaxine in a dose of $75 \mathrm{mg}$ and multivitamins on his own which helped him a lot for a period of two years to manage his job and postgraduate entrance exams without any major shift in mood.

The patient wanted to pursue his career as psychiatrist but senior psychiatrists refused to take him as their trainee after knowing the disease. Then, the patient started M. Phil Physiology but he could not bear the undue stress and again fell into depression despite of taking $75 \mathrm{mg}$ of tablets venlafaxine and multivitamins. Again, the depression was followed by hypomania due to which he had to freeze M. Phil for a period of ten months. During this period when the patient was free, he started studying about his disease.

The history of patient revealed that he suffered from lot of diseases from his early childhood.

At the end of first year of life, he suffered from pneumonia and gastroenteritis which continued intermittently, for four years. Later, he had cracked heals and surgery for haemoroids before age of 
six. When he was in class seventh the subject was diagnosed with amblyopia of left eye and for next five years he suffered from on and off oral ulcers. These events may be responsible as triggering factors for BPD. ${ }^{4}$

Finally, the patient who was a qualified doctor now gambled to take $10 \mathrm{mg}$ of tablet methylphenidate along with $50 \mathrm{mg}$ tablet lamotrigene and 75 mg sustained released tablet venlafaxine for treatment of depression. This regimen proved to be a miracle for him and in next two years he had been absolutely normal without any episode of depression or mania.

The patient got married to a lady doctor who forced him to stop tablet methylphenidate from the above regimen after consulting with professor of psychiatry. After six months, his father died, he switched to severe depression again and the salient feature was deviation towards religion, dryness of mouth and anxiety, which ended in hypomania and the professor of psychiatry advised him with tablet quetiapine in a dose of $200 \mathrm{mg}$ at night and $30 \mathrm{mg}$ of tablet aripiprazole in divided doses. Moreover, $15 \mathrm{mg}$ of tablet procyclidine, and $200 \mathrm{mg}$ of tablet lamotrigine was also prescribed in divided doses.

The patient refused to take the above regimen and started $50 \mathrm{mg}$ of tablet quetiapine at night, $200 \mathrm{mg}$ of tablet lamotrigine and $10 \mathrm{mg}$ of tablets procyclidine in divided doses. The subject is quite normal on above regimen. However, there is severe dryness of mouth and mild tremors in the hands. Moreover, there is lot of drowsiness and dizziness and the subject is trying to concentrate and wake up using tea and coffee and other dietary modifications. The subject is again thinking to use methylphenidate but there are no clinical trials in its favor.

\section{DISCUSSION}

Considerable overlap in diagnostic criteria and shared psychopathologic symptoms in attention deficit hyperactivity disorder (ADHD) and borderline personality disorder over extensive literature search have motivated me to write this case report. Longitudinal studies have shown that
BPD is frequently diagnosed in adult patients who had been diagnosed with ADHD in childhood. The question of whether ADHD and BPD randomly co-occur as comorbidities, have similar origins or share common pathological mechanisms remains unresolved. Some authors suggest that ADHD contributes to the development of BPD via various mechanisms, and therefore consider it a risk factor for later BPD development. ${ }^{5}$

Recent studies reported symptomatic overlap between attention deficit hyperactivity disorder (ADHD) and borderline personality disorder (BPD). Methylphenidate (MPH) is the most efficient treatment for ADHD. Golubchik P. SJ et al tested the efficacy and tolerability of MPH treatment in adolescent females who met the DSM-IV criteria for both disorders. Fourteen BPD/ ADHD female adolescents aged 14-19 years were treated with $\mathrm{MPH}$ for 12 weeks, targeting ADHD, BPD symptoms, and aggressive behavior, as rated by Clinical Global Impression-Severity (CGI-S) and ADHD-rating scale (ADHD-RS) scale for BPD and aggressive behavior severity. ${ }^{5,6}$

Attention deficit hyperactivity disorder (ADHD) is frequently accompanied with borderline personality disorder (BPD). However, few studies have examined how comorbid BPD-ADHD patients, treated with and without methylphenidate (MPH), respond to psychotherapy compared to non-comorbid BPD patients. In this perspective, Matthies SD, Philipsen A used a naturalistic study to compare, during a month-long intensive dialectical behavior therapy (DBT), the clinical course of BPD patients and comorbid BPD$A D H D$ patients who were treated or untreated with MPH. Out of the 158 BPD patients recruited, 59 had adult ADHD as a comorbidity; among these, 29 underwent a treatment with MPH or des-methylphenidate, while the 30 others did not. $\mathrm{MPH}$ treatment was given non-randomly and only when ADHD was considered to be hampering the capacity of the subjects to follow the therapy. Patients completed the following forms upon admission and after 1 month of treatment: the adult ADHD Self-Report Scale (ASRS v.1.1), the Barratt Impulsiveness Scale (BIS-10), the State-Trait Anger Expression (STAXI), the Beck Depression 
Inventory II (BDI-II), and the Beck Hopelessness Scale. At baseline, comorbid BPD-ADHD patients showed significantly higher impulsiveness than BPD patients. In the entire sample, there was a significant decrease in all dimensions ranging from small to large effect sizes during the 4-week intensive DBT. BPD-ADHD patients who were undergoing $\mathrm{MPH}$ treatment showed a significantly improved response to DBT treatment for Trait-State Anger scores, motor impulsiveness, depression severity, and ADHD severity, when compared to those without stimulant medication. The study by Matthies SD, Philipsen A outlines the importance of systematically screening BPD patients for ADHD, since a MPH based treatment will improve the symptoms of patients who are comorbid for BPD and ADHD. ${ }^{5}$ Due to the nonrandom allocation of subjects, more severely affected patients were more readily placed on $\mathrm{MPH}$. This suggests that the more severe the ADHD symptoms, the greater the chance for the patient of being treated. During hypomania there is extreme high vigilance and power of abstract thinking as seen in my subject which is consistent with other studies.

\section{OBJECTIVE}

The purpose of this case report is to describe the role of tablet methylphenidate taken in morning on the treatment of Bipolar depression which is a least studied drug for said purpose.

\section{Consent}

The subject has consented to publish his case report.

\section{CONCLUSIONS}

Methylphenidate may be used in combination with other SSRI's and mood stabilizer in treatment of bipolar depression. The report is written so that methylphenidate may be allowed in the treatment of bipolar depression, if patient feels better with it.

\section{LIMITATIONS}

These are the self-experiences of the subject and may not be applicable to other BPD patients.

\section{Future prospects}

Long term prospective; anterograde and retrograde longitudinal studies may be carried out to study the role of methylphenidate in treatment of BPD.

\section{AKNOWLEGEMENTS}

I am extremely thankful to my supervisor of $M$. Phil, Brig Muhammad Mazhar Hussain, Professor and HoD Physiology, Fazaia Medical College, Islamabad, Pakistan, and Muhammad Ayub, Professor and HoD Physiology, AJK Medical College, Muzaffarabad for their continuous support and encouragement. I am extremely thankful to my parents, siblings and my wife for their support and care.

\section{Copyright(c) 15 July, 2017.}

\section{REFERENCES}

1. De Sousa RT, Machado-Vieira R, Zarate CA, Jr., Manji HK. Targeting mitochondrially mediated plasticity to develop improved therapeutics for bipolar disorder. Expert opinion on therapeutic targets. 2014;18(10):113147. Epub 2014/07/25.

2. Cumyn L, French L, Hechtman L. Comorbidity in adults with attention-deficit hyperactivity disorder. Can J Psychiatry. 2009;54.

3. Diogo R. Lara LWB, Luciano R. Munari. Antidepressant, mood stabilizing and procognitive effects of very low dose sublingual ketamine in refractory unipolar and bipolar depression. Int J Neuropsychopharmacol. 2013;16(9): 2111-7.

4. Ball JS, Links PS. Borderline personality disorder and childhood trauma: evidence for a causal relationship. Curr Psychiatry Rep. 2009;11.

5. Matthies SD, Philipsen A. Common ground in Attention Deficit Hyperactivity Disorder (ADHD) and Borderline Personality Disorder (BPD)-review of recent findings. Borderline personality disorder and emotion dysregulation. 2014;1:3. Epub 2014/01/01.

6. Golubchik P1 SJ, Zalsman G, Weizman A. Methylphenidate in the treatment of female adolescents with cooccurrence of attention deficit/ hyperactivity disorder and borderline personality disorder: a preliminary open-label trial. Int Clin Psychopharmacol. $2008 \mathrm{Jul}$;(4):228-31. doi: 10.1097/ YIC.0b013e3282f94ae2PMID: 18446088. Int Clin Psychopharmacol. 2008 Jul;23(4):228-31.

7. Arntz A, Appels C, Sieswerda S. Hypervigilance in borderline disorder: a test with the emotional Stroop paradigm. J Pers Disord. 2000;14. 


\title{
CORRECTION
}

The amendment of the Professional Vol: 24, No.08 (Prof-3958) titled: "Dengue rapid diagnostic tests; evaluation of utility in a tertiary care hospital" on page 1216 is as under;

\section{INCORRECT}

Aneela Altaf Kidwai ${ }^{1}$, Jamal Ara $^{2}$, Samina Ghaznawi ${ }^{3}$, Shumaila Abdul Rasheed ${ }^{4}$, Saleemullah Paracha ${ }^{5}$, Tahir Hussain $^{6}$

\section{FCPS}

Department of Medicine, Abbasi Shaheed Hospital Karachi, Pakistan.

2. FCPS

Department of Medicine, Abbasi Shaheed Hospital Karachi, Pakistan.

3. MCPS, FCPS

Department of Medicine, Abbasi Shaheed Hospital Karachi, Pakistan.

4. (Post Graduate Trainee) Department of Medicine, Abbasi Shaheed Hospital Karachi, Pakistan.

5. MRCP (UK), FRCP (Edin), FCPS

Department of Medicine, Abbasi Shaheed Hospital Karachi, Pakistan.

6. MRCP (U.K) Department of Medicine, Abbasi Shaheed Hospital Karachi, Pakistan.

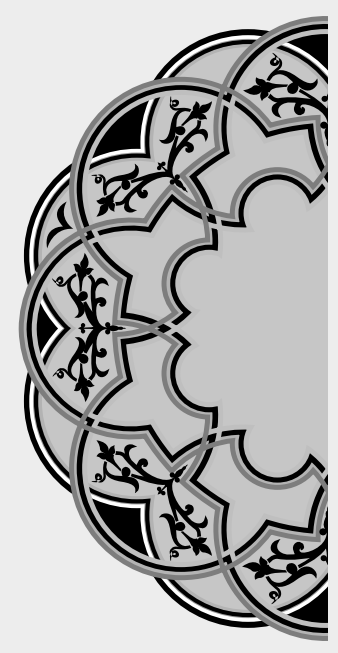

\section{CORRECT}

Aneela Altaf Kidwai ${ }^{1}$, Jamal Ara ${ }^{2}$, Samina Ghaznawi ${ }^{3}$, Shumaila Abdul Rasheed ${ }^{4}$, Saleemullah Paracha ${ }^{5}$, Tahir Hussain $^{6}$

1. FCPS

Associate Professor of Medicine

Abbasi Shaheed Hospital

Karachi, Pakistan.

2. FCPS

Assistant Professor of Medicine

Abbasi Shaheed Hospital

Karachi, Pakistan.

3. MCPS, FCPS

Assistant Professor of Medicine Liaquat National Hospital \& Medical

College.

4. (Post Graduate Trainee) Department of Medicine, Abbasi Shaheed Hospital Karachi, Pakistan.

5. MRCP (U.K)

Professor of Medicine Abbasi Shaheed Hospital

Karachi, Pakistan.

6. MRCP (UK), FRCP (Edin), FCPS

Professor of Medicine Abbasi Shaheed Hospital Karachi, Pakistan

\section{"By failing to prepare, you are preparing to fail."}

\author{
Benjamin Franklin
}

\section{AUTHORSHIP AND CONTRIBUTION DECLARATION}

\begin{tabular}{c|c} 
Sr. \# & Author-s Full Name \\
\hline 1 & Muhammad Sajid Mehmood
\end{tabular}

Contribution to the paper

Author $=$ s Signature
Stadied the case over long time and writer of this case report. 